\title{
Evaluation of Central Blood Pressure in an Asian Population: Comparison between Brachial Oscillometry and Radial Tonometry Methods
}

\author{
Satoshi Hoshide Takahiro Komori Yukiyo Ogata Kazuo Eguchi \\ Kazuomi Kario \\ Division of Cardiovascular Medicine, Department of Medicine, Jichi Medical University \\ School of Medicine, Shimotsuke, Japan
}

\author{
Keywords \\ Central blood pressure $\cdot$ Asian population
}

\begin{abstract}
Background: New devices have recently been developed using various features of the waveform derived from a brachial cuff for noninvasive estimation of central systolic blood pressure (SBP). Central SBP estimated from brachial oscillometry has never been compared with that estimated from radial tonometry in a Japanese population. Subjects and Methods: We recruited 155 Japanese volunteers (mean age $58 \pm 16$ years, range 18-99 years; 66.5\% women) and estimated their central SBP using brachial oscillometry (Mobil-O-Graph) or radial tonometry (SphygmoCor). Results: The mean (standard deviation) peripheral SBP and central SBP measured with brachial oscillometry was $128 \pm 18 \mathrm{~mm} \mathrm{Hg}$ and $118 \pm 16 \mathrm{~mm} \mathrm{Hg}$, respectively, while the central SBP estimated using radial tonometry was $119 \pm 18 \mathrm{~mm} \mathrm{Hg}$. The mean (standard deviation) difference in estimated central SBP between brachial oscillometry and radial tonometry was $0.36 \pm 5.9 \mathrm{~mm} \mathrm{Hg}$, and the central SBPs estimated using these devices were strongly correlated ( $r=0.946$, intraclass correlation coefficient $=0.940, p<0.001)$. Conclusion: Central SBP estimated using brachial oscillometry was similar to that estimated from radial tonometry in a Japanese population.


Hoshide et al.: Central Blood Pressure in Brachial and Radial Methods

\section{Introduction}

In clinical and research settings, central blood pressure (BP) is often measured using noninvasive approaches. Many studies have reported that central systolic blood pressure (SBP) may be a better predictor of cardiovascular events than conventional brachial SBP [1-3]. The SphygmoCor device is widely used for the noninvasive estimation of central hemodynamic variables $[2,4,5]$. However, it requires operator-dependent acquisition of the initial waveform for measuring central BP, and substantial operator training is needed for maintaining accuracy. Despite the complexity of this method, the SphygmoCor is often used in clinical practice to evaluate central hemodynamic variables because substantial SphygmoCor data on central BP from various races, such as Asian [6], African [7], and European [8], are available, and the SphygmoCor approach has therefore often been widely applied as a prognostic factor of intermediate and hard outcomes in various races [2, 4, 5].

Recently, new devices have been developed using various features of the waveform derived from a brachial cuff for noninvasive estimation of central SBP. One such device is the Mobil-O-Graph. Although several studies have compared central BP estimated using the SphygmoCor and the Mobil-O-Graph, to our knowledge most studies were conducted in Western populations $[9,10]$. In the present study, we aimed to compare central BP estimated using the SphygmoCor with that estimated using the Mobil-0-Graph in a Japanese population.

\section{Methods}

All subjects volunteered to participate in this study and provided informed consent. Initially, central and peripheral SBP were measured using the Mobil-O-Graph (I.E.M. GmbH, Stolberg, Germany) on the left arm with the subject in the seated position after resting for at least $10 \mathrm{~min}$. Subsequently, SphygmoCor (AtCor Medical Pty Ltd, West Ryde, Australia) measurements were taken with the subject in the seated position. The SphygmoCor device was calibrated on the basis of brachial BP readings obtained using the Mobil-O-Graph. Measurements of radial artery waveforms (10 s of wave data were acquired) were sequentially assessed by tonometry with the SphygmoCor measurement on the left arm of each participant following a break of 3 min after the measurement of the Mobil-0-Graph. The quality of the recordings was maintained by discarding all SphygmoCor measurements with an operator index $<80$. Only one reading per device was used in this study.

\section{Statistical Analysis}

All measurements are presented as mean \pm standard deviation. Pearson's correlation coefficients were used to assess the association between variables. The relationship between central SBP recordings obtained using different devices was assessed from the intraclass correlation coefficients using the two-way random model of absolute agreement. Differences between central and peripheral SBP were assessed using the paired Student $t$ test. Differences with a $p$ value $<0.05$ were considered statistically significant. Data were analyzed using Bland and Altman plots. These are mainly helpful to represent data graphically and to investigate the agreement of measurements obtained using the different methods. The SPSS version 19.0J software (SPSS, Chicago, IL, USA) was used for statistical analysis.

\section{Results}

We enrolled 155 volunteers in this study. Their detailed baseline characteristics are shown in Table 1. The peripheral SBP and diastolic BP measured using the Mobil-0-Graph were $128 \pm 18$ and $79 \pm 12 \mathrm{~mm} \mathrm{Hg}$, respectively. The mean central SBP measured using the Mobil-O-Graph was $118.4 \pm 16.1 \mathrm{~mm}$ Hg. The mean central SBP measured using the SphygmoCor was $118.8 \pm 18.1 \mathrm{~mm}$ Hg. The average difference was $-0.36 \pm 5.94 \mathrm{~mm}$ Hg. The mean central SBP measured using the Mobil-O-Graph was correlated with that measured using the 
Fig. 1. Scatter plot of the association between central systolic blood pressure (SBP) of the SphygmoCor and central SBP of the Mobil-O-Graph.

Table 1. Baseline characteristics of the 155 volunteers

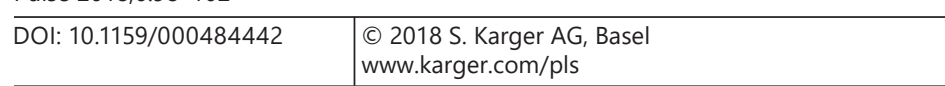

Hoshide et al.: Central Blood Pressure in Brachial and Radial Methods

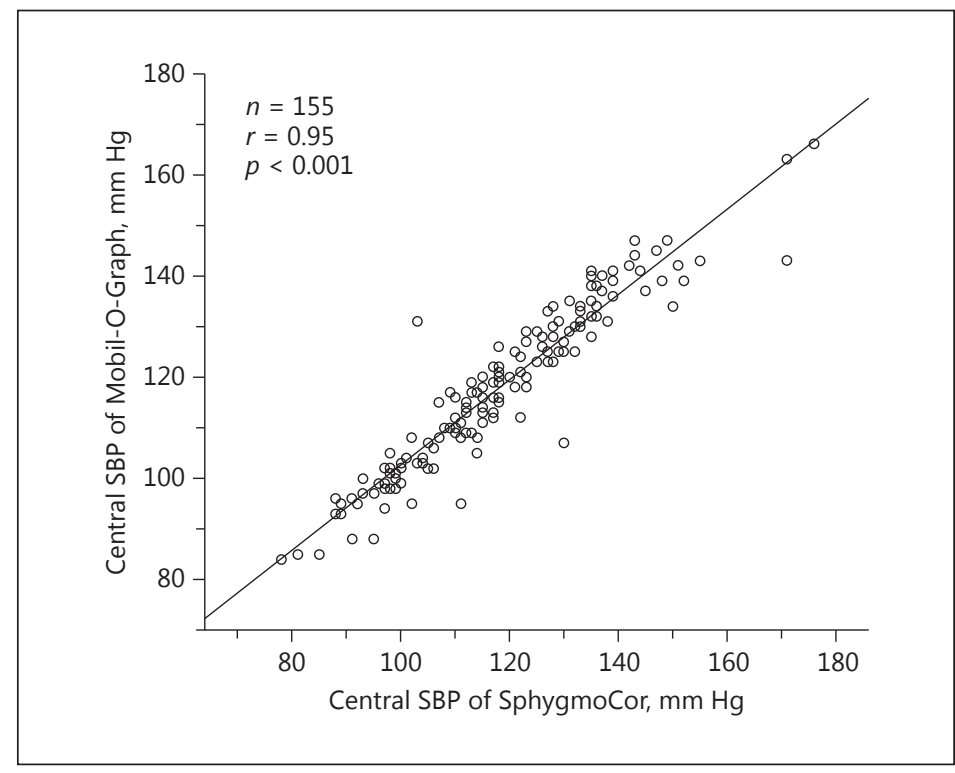

\begin{tabular}{lc}
\hline Age, years & $58 \pm 16$ \\
Male, \% & 33.5 \\
Height, m & $1.60 \pm 0.08$ \\
Weight, kg & $58 \pm 11$ \\
Body mass index & $22.6 \pm 3.2$ \\
Diabetes, \% & 3.9 \\
Hyperlipidemia, \% & 12.3 \\
Hypertension, \% & 14.8 \\
Systolic blood pressure, mm Hg & $128 \pm 18$ \\
Diastolic blood pressure, mm Hg & $79 \pm 12$ \\
Heart rate, bpm & $75 \pm 11$ \\
\hline
\end{tabular}

SphygmoCor $(r=0.95, p<0.001)$ (Fig. 1). The mean intraclass correlation coefficient for the different methods of measuring central SBP was 0.940 (95\% confidence interval 0.918-0.956). The Bland-Altman plots (Fig. 2) showed good agreement (mean difference $0.36 \mathrm{~mm} \mathrm{Hg}$ ).

\section{Discussion}

Our findings show that the Mobil-O-Graph, which uses brachial oscillometric BP for noninvasive estimation of central BP, provides similar central SBP findings as the SphygmoCor device in an Asian population.

In the present study, we used the SphygmoCor device as a reference and compared its readings with those of the Mobil-O-Graph device. We found that the central SBP readings of the SphygmoCor were slightly higher than those of the Mobil-O-Graph, although the difference was $<0.5 \mathrm{~mm} \mathrm{Hg}$. In their study on 100 individuals in the West, Weiss et al. [10] already reported that the Mobil-0-Graph device is as effective for measuring central BP as the SphygmoCor device. They showed that central SBP computed using the SphygmoCor was slightly higher than that computed using the Mobil-O-Graph (124.3 \pm 16.8 vs. $123.7 \pm 15.7 \mathrm{~mm} \mathrm{Hg}$ ), and the difference in average central SBP was $0.5 \mathrm{~mm} \mathrm{Hg}$. Our findings agree with those of previous reports on the Western population. The Association for the Advancement of Medical 
Fig. 2. Bland-Altman plots of central systolic blood pressure (SBP) of the SphygmoCor and the MobilO-Graph.

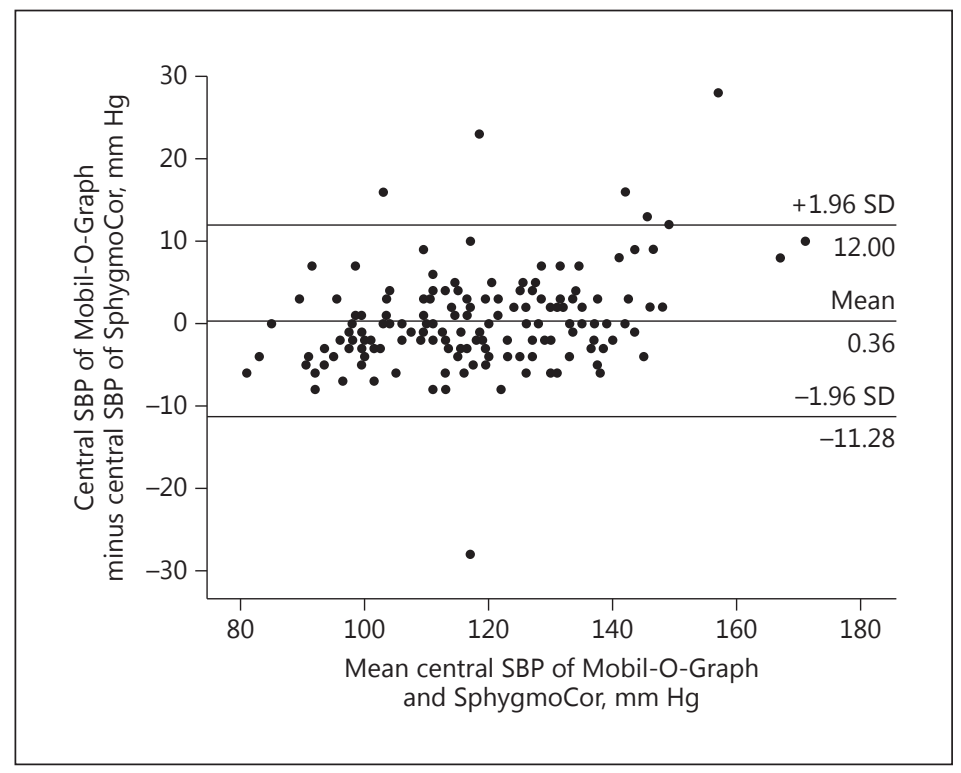

Instrumentation stated that the mean difference and standard deviation for comparison of the two methods should be 5 and $8 \mathrm{mmHg}$, respectively [11]. Our findings were within the limits of this recommendation.

A limitation of the present study is that we did not validate the findings against invasive central BP measurement. Although invasive studies are not easy and ethically acceptable and are impossible to perform on many subjects, previous studies have reported that the values of central BP calculated using the ARCSolver algorithm and the Mobil-O-Graph were similar to those measured invasively in 30 patients [9]. The invasive study also found that the mean difference in central SBP between the ARCSolver algorithm and the SphygmoCor device was $0.5 \mathrm{~mm} \mathrm{Hg}$. The second limitation of the present study was that we used volunteers and not randomly sampled individuals. These volunteers were self-selected and are not necessarily representative of the general population. Lastly, the number of subjects included in this study might appear to be relatively small. However, when power analysis for a linear regression was conducted to determine a sufficient sample size using an alpha of 0.05 , a power of 0.80 , and a medium effect size of 0.3 , the desired sample size was 84 subjects $[12,13]$.

In conclusion, the Mobil-O-Graph, which uses brachial oscillometric BP for noninvasive estimation of central BP, might be clinically used in Asian populations. If our observations can be confirmed in other populations, central BP could be easily and routinely measured in daily practice without operator-dependent acquisition. Moreover, since the Mobil-0-Graph can monitor 24-h central BP profiles, future studies are needed to examine changes in 24-h central $\mathrm{BP}$ readings with antihypertensive treatment and the association between 24-h central BP values and organ damage and clinical outcomes.

\section{Disclosure Statement}

K. Kario has received research grants from Teijin Pharma Limited, Novartis Pharma K.K., Takeda Pharmaceutical Co., Ltd., Omron Healthcare Co., Ltd., Fukuda Denshi, and honoraria from Mochida Pharmaceutical Co., Ltd., Takeda Pharmaceutical Co., Ltd., Daiichi Sankyo Co., Ltd., and Sumitomo Dainippon Pharma Co., Ltd. The other authors have no disclosures. 


\begin{tabular}{l|l}
\hline DOI: $10.1159 / 000484442$ & $\begin{array}{l}\text { @ } 2018 \text { S. Karger AG, Basel } \\
\text { www.karger.com/pls }\end{array}$ \\
\hline
\end{tabular}

Hoshide et al.: Central Blood Pressure in Brachial and Radial Methods

\section{References}

1 Pini R, Cavallini MC, Palmieri V, Marchionni N, Di Bari M, Devereux RB, Masotti G, Roman MJ: Central but not brachial blood pressure predicts cardiovascular events in an unselected geriatric population: the ICARe Dicomano Study. J Am Coll Cardiol 2008;51:2432-2439.

-2 Roman MJ, Devereux RB, Kizer JR, Lee ET, Galloway JM, Ali T, Umans JG, Howard BV: Central pressure more strongly relates to vascular disease and outcome than does brachial pressure: the Strong Heart Study. Hypertension 2007;50:197-203.

3 Vlachopoulos C, Aznaouridis K, O’Rourke MF, Safar ME, Baou K, Stefanadis C: Prediction of cardiovascular events and all-cause mortality with central haemodynamics: a systematic review and meta-analysis. Eur Heart J 2010;31:1865-1871.

-4 Williams B, Lacy PS, Thom SM, Cruickshank K, Stanton A, Collier D, Hughes AD, Thurston H, O'Rourke M: Differential impact of blood pressure-lowering drugs on central aortic pressure and clinical outcomes: principal results of the Conduit Artery Function Evaluation (CAFE) study. Circulation 2006;113:1213-1225.

5 Wang KL, Cheng HM, Sung SH, Chuang SY, Li CH, Spurgeon HA, Ting CT, Najjar SS, Lakatta EG, Yin FC, Chou P, Chen $\mathrm{CH}$ : Wave reflection and arterial stiffness in the prediction of 15-year all-cause and cardiovascular mortalities: a community-based study. Hypertension 2010;55:799-805.

6 6 Li Y, Staessen JA, Li LH, Huang QF, Lu L, Wang JG: Reference values for the arterial pulse wave in Chinese. Am J Hypertens 2008;21:668-673.

-7 Shiburi CP, Staessen JA, Maseko M, Wojciechowska W, Thijs L, van Bortel LM, Woodiwiss AJ, Norton GR: Reference values for SphygmoCor measurements in South Africans of African ancestry. Am J Hypertens 2006; 19:40-46.

8 Wojciechowska W, Staessen JA, Nawrot T, Cwynar M, Seidlerova J, Stolarz K, Gasowski J, Ticha M, Richart T, Thijs L, Grodzicki T, Kawecka-Jaszcz K, Filipovsky J: Reference values in white Europeans for the arterial pulse wave recorded by means of the SphygmoCor device. Hypertens Res 2006;29:475-483.

-9 Weber T, Wassertheurer S, Rammer M, Maurer E, Hametner B, Mayer CC, Kropf J, Eber B: Validation of a brachial cuff-based method for estimating central systolic blood pressure. Hypertension 2011;58:825-832.

10 Weiss W, Gohlisch C, Harsch-Gladisch C, Tolle M, Zidek W, van der Giet M: Oscillometric estimation of central blood pressure: validation of the Mobil-O-Graph in comparison with the SphygmoCor device. Blood Press Monit 2012;17:128-131.

11 White WB, Berson AS, Robbins C, Jamieson MJ, Prisant LM, Roccella E, Sheps SG: National standard for measurement of resting and ambulatory blood pressures with automated sphygmomanometers. Hypertension 1993;21:504-509.

12 Cohen J: Statistical Power Analysis for the Behavioral Sciences, ed 2. Hillsdale, Lawrence Erlbaum Associates, 1988.

13 Faul F, Erdfelder E, Buchner A, Lang AG: Statistical power analyses using G*Power 3.1: tests for correlation and regression analyses. Behav Res Methods 2009;41:1149-1160. 